\title{
Cholesterol in islet dysfunction and type 2 diabetes
}

\author{
Liam R. Brunham, ${ }^{1}$ Janine K. Kruit, ${ }^{1}$ C. Bruce Verchere, ${ }^{2}$ and Michael R. Hayden'
}

${ }^{1}$ Department of Medical Genetics and 2Department of Pathology and Laboratory Medicine, Centre for Molecular Medicine and Therapeutics, Child and Family Research Institute, University of British Columbia, Vancouver, British Columbia, Canada.

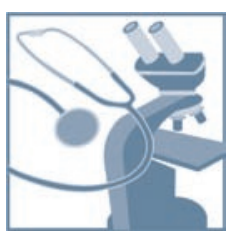

Type 2 diabetes (T2D) frequently occurs in the context of abnormalities of plasma lipoproteins. However, a role for elevated levels of plasma cholesterol in the pathogenesis of this disease is not well established. Recent evidence suggests that alterations of plasma and islet cholesterol levels may contribute to islet dysfunction and loss of insulin secretion. A number of genes involved in lipid metabolism have been implicated in T2D. Recently an important role for ABCA1, a cellular cholesterol transporter, has emerged in regulating cholesterol homeostasis and insulin secretion in pancreatic $\beta$ cells. Here we review the impact of cholesterol metabolism on islet function and its potential relationship to T2D.

Type 2 diabetes (T2D) is characterized by an inability of the endocrine pancreas to secrete sufficient insulin to meet the metabolic demands associated with insulin resistance and obesity. $\beta$ cell dysfunction is a key element in the pathogenesis of this disorder. A reduction in glucose-stimulated insulin secretion is a virtually uniform finding among patients with symptomatic T2D (1). The progression of T2D involves a gradual deterioration in $\beta$ cell function with little or no change in insulin sensitivity (2).

The reasons for $\beta$ cell failure in T2D are not known. The deficit is at least partially a reduction in $\beta$ cell mass (3). However, type 2 diabetic islets are also functionally impaired, as shown by their reduced glucose-stimulated insulin secretion even when corrected for islet mass (4). Several lines of evidence have been advanced to explain the deterioration in insulin secretory output (5). These include $\beta$ cell exhaustion, desensitization to glucose stimulation, deposition of islet amyloid, and lipotoxicity (2).

While elevated levels of plasma cholesterol are a common feature of patients with T2D, the potential role of cholesterol in lipotoxic disease of islets has only recently been explored. Here we review the role of cholesterol metabolism in pancreatic $\beta$ cell function and T2D.

\section{The role of ABCA1 in $\beta$ cell function: in vivo evidence from animal studies}

The most direct evidence for a role of cholesterol metabolism in $\beta$ cell function comes from the study of the ATP-binding cassette transporter, subfamily A, member 1 (ABCA1). ABCA1 is a cellular cholesterol transporter, and mutations in $A B C A 1$ cause Tangier disease (6-8), characterized by an inability to eliminate excess cellular cholesterol, low levels of HDL cholesterol, and increased risk for coronary artery disease (9-11). Study of the specific role of ABCA1 in T2D began with the observation that mice lacking Abca1 globally have significantly impaired glucose tolerance (12). In contrast, insulin sensitivity in these mice is unaltered, suggesting a defect in islet mass or function. This was a surprising find-

Nonstandard abbreviations used: GK, glucokinase; LRP, LDL receptor-related protein; LXR, liver X receptor; nNOS, neuronal NO synthase; T2D, type 2 diabetes.

Conflict of interest: The authors have declared that no conflict of interest exists. Citation for this article: J. Clin. Invest. 118:403-408 (2008). doi:10.1172/JCI33296. ing, as ABCA1 had previously been reported to be expressed in the pancreas at only a very low level (13). However, ABCA1 is highly expressed in the islet cells (12).

Mice with selective inactivation of Abca1 in $\beta$ cells have normal plasma cholesterol levels (12), in contrast to mice lacking Abca1 in the liver (14) or intestine (15). However, islets isolated from mice with $\beta$ cell deletion of $A b c a 1$ have significantly elevated levels of total cholesterol, indicating that ABCA1 is essential for efflux to prevent islet lipid accumulation. Islets from these mice are also functionally impaired, as indicated by their reduced glucose-stimulated insulin secretion. In vivo, mice lacking $\beta$ cell $A b c a 1$ have normal fasting glucose levels but display marked impairment in glucose tolerance and have reduced insulin secretion (12).

The important role of $\beta$ cell ABCA1 in glucose homeostasis was further underscored by the finding that rosiglitazone increases $\beta$ cell ABCA1 expression (12). Rosiglitazone is a member of the thiazolidinedione class of medications that acts primarily as an insulin sensitizer via its action on PPAR- $\gamma$ and subsequent transcriptional activation of a number of genes in insulin-responsive tissues. In humans with impaired glucose tolerance, treatment with rosiglitazone reduces the incidence of T2D (16). Rosiglitazone may also have beneficial effects on $\beta$ cell function in humans (17) and $\beta$ cell mass and function in animal models (18). Troglitazone, another thiazolidinedione, improves $\beta$ cell function by lowering the lipid content of cultured islets isolated from fatty Zucker rats (a model of T2D with insulin resistance and progressive $\beta$ cell deterioration) (19).

In wild-type mice challenged with a high-fat diet, rosiglitazone improves glucose tolerance. However, mice lacking $\beta$ cell Abca1 fail to respond to rosiglitazone treatment, indicating that part of the beneficial effect of rosiglitazone on $\beta$ cell function may be mediated by activating ABCA1 (12).

In humans, rosiglitazone use has been associated with a number of negative side effects such as weight gain, edema, and exacerbation of heart failure (20). In addition, rosiglitazone treatment has been reported to increase the risk of myocardial infarction (21) and hip fractures (22). Targeting ABCA1 expression or activity directly, downstream of PPAR- $\gamma$, may therefore offer therapeutic advantages compared with the thiazolidinediones, enhancing the potentially beneficial effects of increased 


\section{Table 1}

Genes involved in cholesterol metabolism that impact T2D

$\begin{array}{lll}\text { Gene } & \text { Effect } & \text { Reference } \\ A B C A 1 & \text { Variants have been associated with T2D across multiple ethnic groups } & 47,56,57 \\ L X R & \text { Transcriptional activator of } A B C A 1 ; \text { genetic deletion of } L X R \beta \text { results in impaired glucose tolerance and lipid accumulation in islets } & 26 \\ L D L R & \text { Identified in a whole-genome association study of diabetes } & 52 \\ L R P 5 & \text { Mice lacking } L r p 5 \text { have elevated plasma cholesterol levels and impaired insulin secretion from islets } & 31 \\ L R P 6 & \text { Carriers of a mutation in } L R P 6 \text { have elevated plasma } L D L \text { cholesterol levels and an increased risk of developing T2D } & 30 \\ S C D 1 & \text { Mice lacking } S c d 1 \text { have a subpopulation of islets with elevated islet cholesterol levels and attenuated insulin secretion } & 32\end{array}$

ABCA1 activity in cardiovascular disease (23) and $\beta$ cell function and diabetes (24), while minimizing the negative side effects of PPAR- $\gamma$ activation.

\section{Genetic evidence for a role of cholesterol metabolism in T2D}

Studies of genetic conditions in which cholesterol metabolism is altered in humans and mice provide an additional opportunity to examine the role of cholesterol metabolism in islet function (Table 1). The liver X receptor (LXR) is a nuclear hormone receptor that plays a central role in the regulation of cholesterol metabolism and is a major transcriptional activator of $A B C A 1$ (25). $L X R \beta^{-/-}$mice have reduced $A b c a 1$ mRNA levels in islets (26). This is associated with accumulation of neutral lipids in islets, impaired glucose tolerance, and reduced insulin secretion (26). Similarly, activation of LXR in transformed $\beta$ cells results in strong induction of ABCA1 expression and increases insulin secretion (27).

Members of the LDL receptor-related protein (LRP) family have also been implicated in regulation of cholesterol homeostasis and $\beta$ cell function. LRP5 and LRP6 are coreceptors in the Wnt signaling pathway, a pathway involved in normal development and disease $(28,29)$. Mutations in LRP6 were recently described in a family with early coronary artery disease and metabolic risk factors (30). Carriers of the LRP6 mutation have increased LDL cholesterol and triglyceride levels, significantly increased fasting blood glucose, and increased risk for T2D. Of the 15 carriers studied, 11 had T2D (77\%), while all 5 unaffected family members had normal levels of LDL cholesterol and none had T2D.

Another member of the LRP family, LRP5 is required for normal cholesterol and glucose metabolism in mice (31). $\mathrm{rp}^{-1-}$ mice have increased plasma cholesterol levels upon high-fat feeding, due to decreased hepatic clearance of chylomicron remnants. On a standard diet, $L r p 5^{-1-}$ mice have markedly impaired glucose tolerance. Islets isolated from $\mathrm{Lrp5}^{-/-}$mice have impaired glucose-stimulated insulin secretion coinciding with reduced levels of intracellular ATP and calcium in response to glucose.

Stearoyl-CoA desaturase is an enzyme that catalyzes the introduction of a double bond into saturated fatty acids to generate monounsaturated fatty acids. On the diabetogenic obese $o b / o b$ BTBR background, $S c d 1^{-/-}$mice have more severe hyperglycemia (32). $S c d 1^{-1-} ; o b / o b$ BTBR mice have reduced insulin secretion following a glucose challenge, and about half of the islets from these mice were reported to have an abnormally small appearance, impaired insulin secretion, and markedly increased levels of cholesterol as well as fatty acids $(32,33)$. These mutations in various genes provide additional support for the concept that genetic alterations leading to raised plasma cholesterol can be associated with increased risk for developing T2D.

\section{In vitro studies investigating the relationship and mechanism between cholesterol and $\beta$ cell function}

Lipoprotein receptors, in particular the LDL receptor and LRP are expressed in mouse islets (34), suggesting that cholesterol may be taken up by islets via the classic receptor-mediated endocytosis pathway (35). Atherogenic lipoproteins such as LDL and VLDL induce apoptotic death in isolated islets and transformed $\beta$ cell lines $(34,36,37)$. This effect is blocked by HDL (34). These studies suggest that cholesterol loading of islets may be directly cytotoxic. Such a concept is consistent with studies in macrophages showing that free cholesterol induces apoptosis by causing stiffening of the ER membrane, with subsequent induction of the unfolded protein response (38).

Oxidized LDL reduces glucose-stimulated insulin secretion in the clonal hamster $\beta$ cell line, HIT-T15 (39). In contrast, native or acetylated LDL does not reduce insulin secretion. Similarly, oxidized LDL reduces both insulin content and preproinsulin mRNA expression in $\beta$ cell lines and isolated islets (40) and also increases $\beta$ cell apoptosis. Treatment of the cells with inhibitors of the JNK pathway prevent this effect of LDL, suggesting that oxidized LDL reduces insulin expression by activating the JNK pathway. Incubation of cells with HDL blocks the ability of LDL to reduce insulin expression and induce apoptosis.

Cholesterol may directly impair $\beta$ cell function and glucosestimulated insulin secretion (40). Elevating islet cholesterol levels, either in mice lacking Apoe on the diabetogenic obese $o b / o b$ background or by directly overloading transformed $\beta$ cell lines with cholesterol, reduces glucose-stimulated insulin secretion. This is consistent with the reduction in insulin secretion and elevation in islet cholesterol in mice lacking $\beta$ cell Abca1 (12), suggesting a direct effect of cholesterol in reducing $\beta$ cell function. Conversely, lowering $\beta$ cell cholesterol levels with methyl- $\beta$-cyclodextrin (40, 41 , which depletes membrane cholesterol, or with a statin (40) increases insulin secretion.

Collectively these studies indicate that cholesterol is taken up by $\beta$ cells via lipoprotein receptors and that increasing islet cholesterol leads to reduced $\beta$ cell function and, in some studies, increased $\beta$ cell apoptosis. The degree to which these results can be extrapolated to the physiological situation in vivo remains to be determined.

It is essential to determine the mechanisms by which ABCA1 and islet cholesterol impact $\beta$ cell function in order to therapeutically modify these pathways to potentially treat T2D. Some of the potential pathways involved are illustrated in Figure 1. Based on the in vitro studies described above, one could predict that the islet cholesterol accumulation that occurs in the absence of $\beta$ cell ABCA1 would induce apoptosis. However, mice lacking 


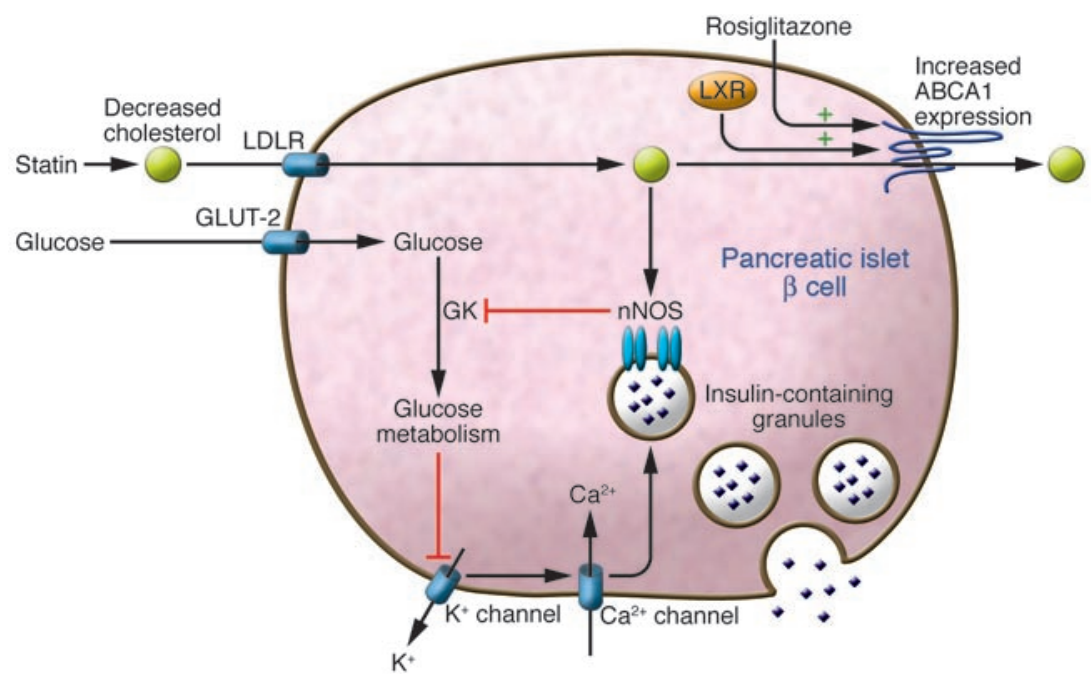

Figure 1

The role of ABCA1 and cholesterol in $\beta$ cell function. The points of potential impact of islet cholesterol levels on insulin secretion are shown. Glucose enters the $\beta$ cell via a glucose transporter (GLUT-2 in rodents), whereupon it is modified by GK in the rate-limiting step in glucose sensing. The subsequent glucose metabolism pathway results in closing of the ATP-sensitive potassium channel, membrane depolarization, calcium influx into the cell via the L-type calcium channel, and exocytosis of insulin-containing granules. Cholesterol is taken up in islets by the LDL receptor. ABCA1 regulates the efflux of cellular cholesterol, and in the absence of $\beta$ cell ABCA1, cellular cholesterol content increases. LXR is a major transcriptional activator of $A B C A 1$, and deletion of $L X R$ results in a similar phenotype to that observed in the absence of $\beta$ cell ABCA1. Elevated $\beta$ cell cholesterol levels may impair insulin secretion by promoting the dimerization of nNOS, which downregulates GK, thus impairing glucose sensing. It is possible that cholesterol also inhibits distal steps in insulin exocytosis and that ABCA1 has other independent effects on insulin secretion. Cholesterollowering drugs such as pravastatin may reduce the incidence of diabetes, potentially by protecting the $\beta$ cell from cholesterol overload. Rosiglitazone, a member of the thiazolidinediones, requires $A B C A 1$ for part of its beneficial effect on $\beta$ cell function, suggesting that targeted therapies that activate $\beta$ cell ABCA1 may have favorable effects on T2D.

$\beta$ cell Abca1 do not appear to have a reduction in $\beta$ cell mass (12). While this finding does not rule out an increased rate of apoptosis, it does suggest that the predominant effect of ABCA1 deficiency and islet cholesterol accumulation in vivo is on $\beta$ cell function rather than mass.

Elevated $\beta$ cell cholesterol may also impair insulin secretion by affecting glucose metabolism upstream (40). Depleting cholesterol from transformed rat (INS-1) $\beta$ cells has been reported to increase glucokinase (GK) activity, whereas culturing cells in cholesterol in the presence of high $(20 \mathrm{mM})$ glucose reduces GK activity. Therefore, GK, which controls the rate-limiting step in $\beta$ cell glucose metabolism, appears to be impacted by $\beta$ cell cholesterol levels. Islet cholesterol levels may also modulate the dimerization state of $\beta$ cell neuronal NO synthase (nNOS) (40), suggesting that excess cholesterol may impair $\beta$ cell glucose metabolism and sensing by increasing the proportion of GK bound to nNOS dimers on the insulin secretory granule membrane, where it is inactive (40).

Cholesterol is known to be a component of both secretory granules and plasma membranes, and cholesterol-rich microdomains in the plasma membrane are thought to be essential for normal exocytosis $(42,43)$. Cholesterol is also enriched in the trans-Golgi network and plays an important role in normal granule formation and trafficking. Lipid rafts rich in cholesterol and sphingolipids are sites of accumulation of soluble $\mathrm{N}$-ethylmaleimide-sensitive fusion protein attachment protein receptors (SNAREs) and other key proteins in exocytosis. Depletion of membrane cholesterol from $\beta$ cells enhances insulin exocytosis by redistributing potassium channels out of lipid rafts (41). It is therefore possible that accumulation of cholesterol in the plasma membrane of $\beta$ cells results in retention of potassium channels in lipid rafts where they are maintained in the open configuration, thus resulting in a reduction in stimulus-coupled insulin secretion.

In mouse models of Smith-Lemli-Opitz syndrome and lathosterolosis, in which cholesterol synthesis is disrupted, there is impaired formation of secretory granules in the endocrine pancreas as well as the anterior pituitary and adrenal medulla, suggesting that cholesterol is essential for the formation of these secretory granules (44). ABCA1-deficient islets have impaired insulin secretion in response to both non-glucose secretagogues as well as glucose (45), yet have elevated insulin content, suggestive of a defect distal in the exocytotic process rather than in islet glucose sensing per se. One parsimonious hypothesis is that while cholesterol is critical for granule fusion, excess cholesterol (for example, in the absence of ABCA1) causes dysregulation of raft formation and impaired exocytosis. An analogous situation may exist with lipoprotein lipase (LPL), which impairs insulin secretion when it is either deleted or overexpressed from $\beta$ cells (46), suggesting that an optimal amount of LPL activity is necessary for normal $\beta$ cell function. An additional possibility is that ABCA1 impairs insulin exocytosis independently of its effects on cellular cholesterol metabolism. Given the accumulating evidence that dysregulated islet cholesterol may play a role in the $\beta$ cell dysfunction of T2D, further study is clearly warranted to elucidate the precise mechanism by which $\beta$ cell cholesterol modulates insulin secretion.

\section{Role of ABCA1 in glucose metabolism in humans}

ABCA1 clearly plays an important role in islet function in the mouse, but what data exist for a role of ABCA1 in glucose metabolism in humans? A number of polymorphisms and mutations in ABCA1 have been associated with T2D across multiple ethnic groups (Table 2). In the Mexican population, the R230C polymorphism is associated with low HDL cholesterol and increased body mass index (BMI) (47). This polymorphism is also significantly associated with the metabolic syndrome, and in a limited sample, individuals with the $\mathrm{C} 230$ allele had a 4.5 -fold increased risk for T2D, a risk that remained significant even after adjusting for HDL cholesterol, BMI, apoA-I levels, and ethnic admixture. In 2 additional larger cohorts of Mexican individuals, the $\mathrm{R} 230 \mathrm{C}$ variant was found to be significantly associated with $\mathrm{T} 2 \mathrm{D}$, particularly early-onset T2D (48). This variant was also associated with higher hemoglobin A1C levels and reduced fasting insulin. Interestingly, of the 10 individuals homozygous for the C230 allele, all 10 had T2D. While functional studies of the 


\section{Table 2}

ABCA1 variants associated with $\mathrm{T} 2 \mathrm{D}$

\begin{tabular}{ll} 
Variant & Effect \\
R230C & Increased risk (4.5-fold) of T2D development in Mexican population \\
K776N & Increased risk $(1.8-f o l d)$ of T2D development in males in Copenhagen Heart Study \\
R1615P & Associated with T2D in a heterozygous patient \\
Haplotype block in intron 2 & Increased $(2.5-$ fold $)$ risk of T2D development in Japanese population \\
Exon 4 mutation & Associated with T2D development in a Japanese patient \\
\hline
\end{tabular}

$\mathrm{R} 230 \mathrm{C}$ variant have not been reported, this variant is predicted to impair the function of the ABCA1 protein based on the evolutionary conservation at this site in related proteins (49).

The R230C variant is also seen in the Oji-Cree population and is linked in these subjects to familial low HDL levels (50). Interestingly, the incidence of T2D in the Oji-Cree population is exceptionally high (51), raising the question of whether the R230C variant may predispose to diabetes in this population. Although the R230C allele is very frequent in the Mexican population, it is not found in Caucasians (50). This might explain why $A B C A 1$ was not identified as a risk locus for T2D in 4 recently published genome-wide association studies in primarily Caucasian populations (52-55).

In a Japanese population of T2D patients and normoglycemic controls, an $A B C A 1$ haplotype block in intron 2 was associated with T2D with an odds ratio of 2.5 (56). This association remained significant after adjustment for BMI and triglyceride levels (57). The functional polymorphisms that underlie this association remain to be identified.

At least 73 mutations have been described in the $A B C A 1$ gene, and these are associated with a spectrum of biochemical and clinical phenotypes (58). At least 2 pathogenic $A B C A 1$ mutations have been reported to be associated with T2D in particular patients. One 33-year-old male with T2D and very low HDL cholesterol $(0.26 \mathrm{mmol} / \mathrm{l})$ was found to be heterozygous for a novel R1615P missense mutation in $A B C A 1$ (59). An additional patient with T2D was found to have a mutation in exon 4 of the $A B C A 1$ gene (60).

In the Copenhagen Heart Study, male carriers of the K776N $A B C A 1$ variant had significantly lower HDL cholesterol and an increased risk of cardiovascular disease. The frequency of T2D was nearly double in male carriers $(11 \%)$ compared with non-carriers $(6 \%)$ of this variant (61). Although this result did not reach statistical significance, it suggests that men with the $\mathrm{K} 776 \mathrm{~N}$ variant may be at increased risk of developing T2D.

Patients with T2D have lower levels of ABCA1 mRNA in monocytes compared with nondiabetic control subjects (62). This raises the question of whether a reduction in ABCA1 levels could contribute to the pathogenesis of T2D in the general population. Alternatively, hyperglycemia may itself downregulate ABCA1, a concept supported by the finding that leukocyte $A B C A 1 \mathrm{mRNA}$ levels are inversely correlated with fasting plasma glucose levels in normoglycemic individuals (63). ABCA1 protein levels are reduced by unsaturated FFAs $(64,65)$ and advanced glycosylation end products (AGEs) (66), suggesting that the imbalances associated with the metabolic syndrome, such as elevated fatty acid levels and hyperglycemia, may themselves reduce ABCA1 activity. In adipocytes, ABCA1 levels are downregulated by insulin (67). This suggests that levels of ABCA1 in cells are associated with circulating levels of both glucose and insulin. It is not known whether the reduction in ABCA1 levels in diabetic subjects is cause or consequence of their disease. However, these findings raise the question of whether reduced $\mathrm{ABCA} 1$ activity could predispose to $\mathrm{T} 2 \mathrm{D}$ in the general population. It would be useful to determine whether the changes in $A B C A 1 \mathrm{mRNA}$ levels reflect genetic variation in $A B C A 1$ and whether they have an impact on cholesterol efflux from $\beta$ cells, which could explain the alterations in fasting glucose levels with which they are associated.

Collectively these studies suggest that impaired ABCA1 activity is associated with an increased risk of diabetes. However, the important question of whether Tangier disease - a rare inherited disorder caused by homozygous mutations in $A B C A 1$ and characterized by a severe reduction in HDL levels (68) - itself predisposes to diabetes remains unanswered. Despite four decades of intensive research into Tangier disease, there is a paucity of published data on glucose homeostasis in these patients. However, the fact that diabetes is not commonly reported as a clinical feature of this disorder would suggest that the patients do not frequently develop T2D. One issue in addressing this question is that humans with Tangier disease have significantly reduced total plasma cholesterol, and in particular, LDL cholesterol is reduced by $40 \%-70 \%$ (69). These beneficial reductions in plasma lipids may limit the extent of $\beta$ cell cholesterol accumulation that occurs in the complete absence of ABCA1 and could partially mask a disturbance in glucose homeostasis.

An analogous situation exists in Abca1 total knockout mice, which have very low levels of total plasma cholesterol (70) and may be partially protected from islet cholesterol accumulation. Indeed, the glucose intolerance phenotype is much more severe in Abca1 $\beta$ cell-specific knockout mice, which have higher levels of circulating cholesterol (12). As a consequence, it may be necessary to study humans heterozygous for $A B C A 1$ mutations, with higher levels of total plasma cholesterol, to detect a phenotype. It should be possible with techniques currently available to determine whether patients heterozygous for an $A B C A 1$ mutation have impaired $\beta$ cell function.

Interestingly, even for the transcription factor-7-like 2 (TCF7L2) gene, one of the genes with the strongest genetic evidence for involvement in the pathogenesis of T2D $(71,72)$, the effect on insulin secretion in humans is quite modest (73). This appears to be the case for other genes strongly linked with T2D (74). If a similar situation holds true for ABCA1, it will be necessary to perform detailed phenotypic studies of $\beta$ cell function to accurately characterize the role of ABCA1 in islet function in humans.

Another exception seems to be familial hypercholesterolemia, in which patients have extremely high levels of LDL cholesterol in plasma but are not reported to develop T2D more frequently or at an earlier age than controls. It is interesting to note, however, 


\section{science in medicine}

that all 5 genetic conditions in humans associated with raised LDL cholesterol levels involve a reduction in the number or activity of LDL receptors $(75,76)$. Cholesterol uptake into islets appears to require the presence of $\mathrm{LDL}$ receptors, as it is reduced in islets isolated from mice lacking the LDL receptor (34). This could indicate that familial hypercholesterolemia and related disorders result in very high levels of cholesterol in plasma but a reduced uptake of cholesterol into islets, potentially explaining the finding that islet function does not appear to be impaired under these conditions.

Plasma cholesterol levels are associated with islet cholesterol content in mice (40). A corollary to this is that lowering levels of plasma cholesterol should reduce islet cholesterol levels and thereby reduce the risk of T2D. Indeed this seems to be the case, at least in certain circumstances. In the West of Scotland Coronary Prevention Study (WOSCOPS) trial, plasma LDL cholesterol was lowered by $26 \%$ with pravastatin, and over the following 6 years the risk of developing T2D was reduced by $30 \%(77,78)$. Lipid lowering is also reported to decrease the risk of $\mathrm{T} 2 \mathrm{D}$ after a myocardial infarction (79). However, not all statins have been reported to reduce the risk of T2D $(80,81)$. The reasons for this difference are not known but could relate to the finding that certain statins appear to negatively impact insulin secretion in vitro $(45,82)$, independently from their lipid-lowering effect.

\section{Conclusion}

T2D is amongst the major health crises of the present age. Data from several sources suggest that alterations of plasma and islet cholesterol metabolism may contribute to the pathogenesis of this disease. ABCA1, which regulates islet cholesterol efflux, is essential for normal $\beta$ cell function, and absence of ABCA1 results in islet cholesterol overload and impaired insulin release. Several genetic alterations of cholesterol metabolism are associated with T2D. In vitro studies suggest that increasing islet cholesterol can induce $\beta$ cell death and impairment of insulin secretion, while reducing islet cholesterol enhances insulin secretion. Individuals with T2D frequently have elevated plasma cholesterol levels, and lowering plasma cholesterol may reduce the risk of developing this disease.

Several important questions remain. Do humans with mutations in ABCA1 have impaired $\beta$ cell function? What are the precise mechanisms by which ABCA1 and cholesterol impact $\beta$ cell function? Do certain statins that reduce the incidence of T2D do so via cholesterol lowering? Many of these questions can now be addressed with currently available tools, and their answers may lead us closer to the tantalizing prospect of targeting cholesterol metabolism in order to simultaneously treat diabetes and the number one cause of death of persons with diabetes, namely cardiovascular disease.

\section{Acknowledgments}

This work was supported by grants from the Canadian Institutes of Health Research (CIHR) to C.B. Verchere and M.R. Hayden. L.R. Brunham is supported by a CIHR studentship and is a Michael Smith Foundation for Health Research (MSFHR) doctoral trainee. J.K. Kruit is supported by a Child and Family Research Institute fellowship. C.B. Verchere is an MSFHR Senior Scholar. M.R. Hayden holds a Canada Research Chair in Human Genetics and is a University Killam Professor. Special thanks to Mahmoud Pouladi for expert graphic advice.

Address correspondence to: Michael R. Hayden, Centre for Molecular Medicine and Therapeutics, Child and Family Research Institute, University of British Columbia, 950 West 28th Avenue, Vancouver, British Columbia V5Z 4H4, Canada. Phone: (604) 875-3535; Fax: (604) 875-3819; E-mail: mrh@cmmt.ubc.ca.
1. Perley, M.J., and Kipnis, D.M. 1967. Plasma insulin responses to oral and intravenous glucose: studies in normal and diabetic subjects. J. Clin. Invest. 46:1954-1962.

2. Kahn, S.E. 2001. The importance of beta-cell failure in the development and progression of type 2 diabetes. J. Clin. Endocrinol. Metab. 86:4047-4058.

3. Butler, A.E., et al. 2003. Beta-cell deficit and increased beta-cell apoptosis in humans with type 2 diabetes. Diabetes. 52:102-110.

4. Deng, S., et al. 2004. Structural and functional abnormalities in the islets isolated from type 2 diabetic subjects. Diabetes. 53:624-632.

5. Prentki, M., and Nolan, C.J. 2006. Islet beta cell failure in type 2 diabetes. J. Clin. Invest. 116:1802-1812.

6. Brooks-Wilson, A., et al. 1999. Mutations in ABC1 in Tangier disease and familial high-density lipoprotein deficiency. Nat. Genet. 22:336-345.

7. Rust, S., Rosier, M., et al. 1999. Tangier disease is caused by mutations in the gene encoding ATP-binding cassette transporter 1. Nat.Genet. 22:352-355.

8. Bodzioch, M., et al. 1999. The gene encoding ATPbinding cassette transporter 1 is mutated in Tangier disease. Nat. Genet. 22:347-351.

9. Schaefer, E.J., Zech, L.A., Schwartz, D.E., and Brewer, H.B., Jr. 1980. Coronary heart disease prevalence and other clinical features in familial high-density lipoprotein deficiency (Tangier disease). Ann. Intern. Med. 93:261-266.

10. Clee, S.M., et al. 2000. Age and residual cholesterol efflux affect HDL cholesterol levels and coronary artery disease in ABCA1 heterozygotes. J. Clin. Invest. 106:1263-1270.

11. van Dam, M.J., et al. 2002. Association between increased arterial-wall thickness and impairment in ABCA1-driven cholesterol efflux: an observational study. Lancet. 359:37-42.

12. Brunham, L.R., et al. 2007. Beta-cell ABCA1 influences insulin secretion, glucose homeostasis and response to thiazolidinedione treatment. Nat. Med. 13:340-347.

13. Langmann, T., et al. 1999. Molecular cloning of the human ATP-binding cassette transporter 1 (hABC1): evidence for sterol-dependent regulation in macrophages. Biochem. Biophys. Res. Commun. 257:29-33

14. Timmins, J.M., et al. 2005. Targeted inactivation of hepatic Abca1 causes profound hypoalphalipoproteinemia and kidney hypercatabolism of apoA-I. J. Clin. Invest. 115:1333-1342.

15. Brunham, L.R., et al. 2006. Intestinal ABCA1 directly contributes to HDL biogenesis in vivo. J. Clin. Invest. 116:1052-1062.

16. The DREAM (Diabetes REduction Assessment with ramipril and rosiglitazone Medication) Trial Investigators. 2006. Effect of rosiglitazone on the frequency of diabetes in patients with impaired glucose tolerance or impaired fasting glucose: a randomised controlled trial. Lancet. 368:1096-1105.

17. Cavaghan, M.K., Ehrmann, D.A., Byrne, M.M., and Polonsky, K.S. 1997. Treatment with the oral antidiabetic agent troglitazone improves beta cell responses to glucose in subjects with impaired glucose tolerance. J. Clin. Invest. 100:530-537.

18. Higa, M., et al. 1999. Troglitazone prevents mitochondrial alterations, beta cell destruction, and diabetes in obese prediabetic rats. Proc. Natl. Acad. Sci. U. S. A. 96:11513-11518.

19. Shimabukuro, M., Zhou, Y.T., Lee, Y., and Unger, R.H. 1998. Troglitazone lowers islet fat and restores beta cell function of Zucker diabetic fatty rats. J. Biol. Chem. 273:3547-3550.

20. Home, P.D., et al. 2007. Rosiglitazone evaluated for cardiovascular outcomes - an interim analysis. N. Engl. J. Med. 357:28-38.

21. Nissen, S.E., and Wolski, K. 2007. Effect of rosiglitazone on the risk of myocardial infarction and death from cardiovascular causes. N. Engl. J. Med. 356:2457-2471.

22. Kahn, S.E., et al. 2006. Glycemic durability of rosiglitazone, metformin, or glyburide monotherapy. N. Engl. J. Med. 355:2427-2443.

23. Singaraja, R.R., et al. 2002. Increased ABCA1 activity protects against atherosclerosis. J. Clin. Invest. 110:35-42.

24. Chakravarthy, M.V., and Semenkovich, C.F. 2007. The ABCs of beta-cell dysfunction in type 2 diabetes. Nat. Med. 13:241-242.

25. Zelcer, N., and Tontonoz, P. 2006. Liver X receptors as integrators of metabolic and inflammatory signaling. J. Clin. Invest. 116:607-614.

26. Gerin, I., et al. 2005. LXRbeta is required for adipocyte growth, glucose homeostasis, and beta cell function. J. Biol. Chem. 280:23024-23031.

27. Efanov, A.M., Sewing, S., Bokvist, K., and Gromada, J. 2004. Liver X receptor activation stimulates insulin secretion via modulation of glucose and lipid metabolism in pancreatic beta-cells. Diabetes. 53(Suppl. 3):S75-S78.

28. Pinson, K.I., Brennan, J., Monkley, S., Avery, B.J., and Skarnes, W.C. 2000. An LDL-receptor-related protein mediates Wnt signalling in mice. Nature. 407:535-538.

29. Tamai, K., et al. 2000. LDL-receptor-related proteins in Wnt signal transduction. Nature. 407:530-535. 
30. Mani, A., et al. 2007. LRP6 mutation in a family with early coronary disease and metabolic risk factors. Science. 315:1278-1282.

31. Fujino, T., et al. 2003. Low-density lipoprotein receptor-related protein 5 (LRP5) is essential for normal cholesterol metabolism and glucoseinduced insulin secretion. Proc. Natl. Acad. Sci.U.S. A 100:229-234

32. Flowers, J.B., et al. 2007. Loss of stearoyl-CoA desaturase- 1 improves insulin sensitivity in lean mice but worsens diabetes in leptin-deficient obese mice. Diabetes. 56:1228-1239.

33. Attie, A.D., et al. 2007. Stearoyl-CoA desaturase deficiency, hypercholesterolemia, cholestasis, and diabetes. Nutr. Rev. 65:S35-S38.

34. Roehrich, M.E., et al. 2003. Insulin-secreting betacell dysfunction induced by human lipoproteins. J. Biol. Chem. 278:18368-18375.

35. Brown, M.S., and Goldstein, J.L. 1986. A receptormediated pathway for cholesterol homeostasis. Science. 232:34-47.

36. Cnop, M., Hannaert, J.C., Grupping, A.Y., and Pipeleers, D.G. 2002. Low density lipoprotein can cause death of islet beta-cells by its cellular uptake and oxidative modification. Endocrinology. 143:3449-3453.

37. Grupping, A.Y., et al. 1997. Low density lipoprotein binding and uptake by human and rat islet beta cells. Endocrinology. 138:4064-4068.

38. Yao, P.M., and Tabas, I. 2001. Free cholesterol loading of macrophages is associated with widespread mitochondrial dysfunction and activation of the mitochondrial apoptosis pathway. J. Biol. Chem. 276:42468-42476.

39. Abderrahmani, A., et al. 2007. Human high-density lipoprotein particles prevent activation of the JNK pathway induced by human oxidised low-density lipoprotein particles in pancreatic beta cells. Diabetologia. 50:1304-1314

40. Hao, M., Head, W.S., Gunawardana, S.C., Hasty, A.H and Piston, D.W. 2007. Direct effect of cholesterol on insulin secretion: a novel mechanism for pancreatic beta-cell dysfunction. Diabetes. 56:2328-2338.

41. Xia, F., et al. 2004. Disruption of pancreatic betacell lipid rafts modifies Kv2.1 channel gating and insulin exocytosis. J. Biol. Chem. 279:24685-24691.

42. Salaun, C., James, D.J., and Chamberlain, L.H. 2004. Lipid rafts and the regulation of exocytosis. Traffic. 5:255-264.

43. Wang, Y., Thiele, C., and Huttner, W.B. 2000. Cholesterol is required for the formation of regulated and constitutive secretory vesicles from the transGolgi network. Traffic. 1:952-962.

44. Gondre-Lewis, M.C., et al. 2006. Abnormal sterols in cholesterol-deficiency diseases cause secretory granule malformation and decreased membrane curvature. J. Cell Sci. 119:1876-1885.

45. Ishikawa, M., et al. 2006. Distinct effects of pravastatin, atorvastatin, and simvastatin on insulin secretion from a beta-cell line, MIN6 cells. J. Atheroscler. Thromb. 13:329-335.

46. Pappan, K.L., et al. 2005. Pancreatic beta-cell lipoprotein lipase independently regulates islet glucose metabolism and normal insulin secretion. J. Biol. Chem. 280:9023-9029.

47. Villarreal-Molina, M.T., et al. 2007. The ABCA1 R230C variant affects HDL-cholesterol levels and body mass index in the Mexican population: association with obesity and obesity-related comorbidities.
Diabetes. 56:1881-1887.

48. Villarreal-Molina, M.T., et al. 2007. Association of the ATP-binding cassette transporter A1 R230C variant with early-onset type 2 diabetes in the Mexican population. Diabetes. doi:10.2337/db07-0484.

49. Brunham, L.R., et al. 2005. Accurate prediction of the functional significance of single nucleotide polymorphisms and mutations in the $A B C A 1$ gene. PLoS Genetics. 1:e83.

50. Wang, J., et al. 2000. Common and rare ABCA1 variants affecting plasma HDL cholesterol. Arterioscler. Thromb. Vasc. Biol. 20:1983-1989.

51. Harris, S.B., et al. 1997. The prevalence of NIDDM and associated risk factors in native Canadians. Diabetes Care. 20:185-187.

52. Sladek, R., et al. 2007. A genome-wide association study identifies novel risk loci for type 2 diabetes. Nature. 445:881-885.

53. Zeggini, E., et al. 2007. Replication of genome-wide association signals in UK samples reveals risk loci for type 2 diabetes. Science. 316:1336-1341.

54. Scott, L.J., et al. 2007. A genome-wide association study of type 2 diabetes in Finns detects multiple susceptibility variants. Science. 316:1341-1345.

55. Diabetes Genetics Initiative of Broad Institute of Harvard and MIT, et al. 2007. Genome-wide association analysis identifies loci for type 2 diabetes and triglyceride levels. Science. 316:1331-1336.

56. Daimon, M., et al. 2005. Association of the ABCA1 gene polymorphisms with type $2 \mathrm{DM}$ in a Japanese population. Biochem. Biophys. Res. Commun. 329:205-210.

57. Daimon, M., et al. 2003. Large-scale search of SNPs for type $2 \mathrm{DM}$ susceptibility genes in a Japanese population. Biochem. Biophys. Res. Commun. 302:751-758.

58. Brunham, L.R., Singaraja, R.R., and Hayden, M.R. 2006. Variations on a gene: rare and common variants in ABCA1 and their impact on HDL cholesterol levels and atherosclerosis. Annu. Rev. Nutr. 26:105-129.

59. Saleheen, D., Nazir, A., Khanum, S., Haider, S.R., and Frossard, P.M. 2005. R1615P: A novel mutation in ABCA1 associated with low levels of HDL and type II diabetes mellitus. Int. J. Cardiol. 110:259-260.

60. Saito, M., et al. 2002. Case of familial hypoalphalipoproteinemia, type 2 diabetes mellitus and markedly advanced atherosclerosis with ABCA1 exon 4 minus transcript in macrophages [In Japanese]. Nippon Naika Gakkai Zasshi. 91:2762-2764.

61. Frikke-Schmidt, R., et al. 2005. Mutation in ABCA1 predicted risk of ischemic heart disease in the Copenhagen City Heart Study Population. J. Am. Coll. Cardiol. 46:1516-1520.

62. Forcheron, F., Cachefo, A., Thevenon, S., Pinteur, C., and Beylot, M. 2002. Mechanisms of the triglyceride- and cholesterol-lowering effect of fenofibrate in hyperlipidemic type 2 diabetic patients. Diabetes. 51:3486-3491.

63. Albrecht, C., et al. 2004. Leukocyte ABCA1 gene expression is associated with fasting glucose concentration in normoglycemic men. Metabolism. 53:17-21.

64. Wang, Y., and Oram, J.F. 2005. Unsaturated fatty acids phosphorylate and destabilize ABCA1 through a phospholipase D2 pathway. J. Biol. Chem. 280:35896-35903.

65. Wang, Y., Kurdi-Haidar, B., and Oram, J.F. 2004. LXR-mediated activation of macrophage stearoyl-
CoA desaturase generates unsaturated fatty acids that destabilize ABCA1. J. Lipid Res. 45:972-980.

66. Passarelli, M., et al. 2005. Advanced glycation end product precursors impair ABCA1-dependent cholesterol removal from cells. Diabetes. 54:2198-2205.

67. Sartipy, P., and Loskutoff, D.J. 2003. Expression profiling identifies genes that continue to respond to insulin in adipocytes made insulin-resistant by treatment with tumor necrosis factor-\{alpha\}. J. Biol. Chem. 278:52298-52306.

68. Singaraja, R.R., Brunham, L.R., Visscher, H., Kastelein, J.J., and Hayden, M.R. 2003. Efflux and atherosclerosis: the clinical and biochemical impact of variations in the ABCA1 gene. Arterioscler. Thromb. Vasc. Biol. 23:1322-1332.

69. Schaefer, E.J., et al. 2001. Cholesterol and apolipoprotein B metabolism in Tangier disease. Atherosclerosis. 159:231-236.

70. McNeish, J., et al. 2000. High density lipoprotein deficiency and foam cell accumulation in mice with targeted disruption of ATP-binding cassette transporter-1. Proc. Natl. Acad. Sci. U. S. A. 97:4245-4250.

71. Grant, S.F.A., et al. 2006. Variant of transcription factor 7-like 2 (TCF7L2) gene confers risk of type 2 diabetes. Nat. Genet. 38:320-323.

72. Florez, J.C., et al. 2006. TCF7L2 Polymorphisms and progression to diabetes in the Diabetes Prevention Program. N. Engl. J. Med. 355:241-250.

73. Lyssenko, V., et al. 2007. Mechanisms by which common variants in the TCF7L2 gene increase risk of type 2 diabetes. J. Clin. Invest. 117:2155-2163.

74. Staiger, H. 2007. Polymorphisms within novel risk loci for type 2 diabetes determine beta-cell function. PLOS ONE. 2:e832.

75. Goldstein, J.L., and Brown, M.S. 2001. Molecular medicine. The cholesterol quartet. Science. 292:1310-1312.

76. Horton, J.D., Cohen, J.C., and Hobbs, H.H. 2007. Molecular biology of PCSK9: its role in LDL metabolism. Trends Biochem. Sci. 32:71-77.

77. Shepherd, J., et al. 1995. Prevention of coronary heart disease with pravastatin in men with hypercholesterolemia. N. Engl. J. Med. 333:1301-1308.

78. Freeman, D.J., et al. 2001. Pravastatin and the development of diabetes mellitus: evidence for a protective treatment effect in the West of Scotland Coronary Prevention Study. Circulation. 103:357-362.

79. Mozaffarian, D., et al. 2007. Incidence of new-onset diabetes and impaired fasting glucose in patients with recent myocardial infarction and the effect of clinical and lifestyle risk factors. Lancet. 370:667-675.

80. Collins, R., et al. 2003. MRC/BHF Heart Protection Study of cholesterol-lowering with simvastatin in 5963 people with diabetes: a randomised placebocontrolled trial. Lancet. 361:2005-2016.

81. Sever, P.S., et al. 2003. Prevention of coronary and stroke events with atorvastatin in hypertensive patients who have average or lower-than-average cholesterol concentrations, in the Anglo-Scandinavian Cardiac Outcomes Trial - Lipid Lowering Arm (ASCOT-LLA): a multicentre randomised controlled trial. Lancet. 361:1149-1158.

82. Yada, T., Nakata, M., Shiraishi, T., and Kakei, M. 1999. Inhibition by simvastatin, but not pravastatin, of glucose-induced cytosolic $\mathrm{Ca} 2+$ signalling and insulin secretion due to blockade of L-type $\mathrm{Ca} 2+$ channels in rat islet beta-cells. Br. J. Pharmacol. 126:1205-1213 